\title{
Further Results on Model Structure Validation for Closed Loop System Identification
}

\author{
Wang Jian-hong, Wang Yan-xiang \\ School of Mechanical and Electronic Engineering, Jingdezhen Ceramic Institute, Jingdezhen, China \\ Email address: \\ wangyanxiang1@jci.edu.cn (Wang Yan-xiang) \\ To cite this article: \\ Wang Jian-hong, Wang Yan-xiang. Further Results on Model Structure Validation for Closed Loop System Identification. Advances in \\ Wireless Communications and Networks. Vol. 3, No. 5, 2017, pp. 57-66. doi: 10.11648/j.awcn.20170305.12
}

Received: May 18, 2017; Accepted: June 28, 2017; Published: August 24, 2017

\begin{abstract}
In this paper, further results on the problem of the model structure validation for closed loop system identification are proposed. One probabilistic model uncertainty is derived from some statistical properties of the parameter estimation. The uncertainties bound of the model parameter is constructed in the probability sense by using the inner product form of the asymptotic covariance matrix. Further a new technique for estimating bias and variance contributions to the model error is suggested. One bound described as an inequality corresponds to a condition on the model error. Due to this proposed bound, model structure validation process can be transformed to verify whether the model error obeys this inequality. Finally the simulation example results confirm the identification theoretical results.
\end{abstract}

Keywords: Closed Loop Identification, Model Uncertainty, Model Structure Validity, One Bound

\section{Introduction}

The automatic control system includes two basic structures: one open loop and other closed loop. As there does not exist any feedback in open loop structure, so the plant output affects the input less. And in closed open structure, the error signals coming from the input and feedback output generate one correction action and make the output converge to some given value. The essence of closed loop system is to decrease the error by using the negative feedback function, and correct the deviation from the given value automatically. As the closed loop structure can suppress the errors coming from the internal or external disturbances, so for many industrial production processes, safety and production restrictions are strong reasons for not allowing control experiments in open-loop and the closed loop is most needed in all of our engineering.

Generally two strategies are used to design the controller in closed loop, i.e. model based design and direct data driven design. The primary step of model based design is to construct the plant model and apply this mathematical model in the process of designing controller. Conversely in the direct data driven method, the modeling process is not needed and the controller is directly designed by using the input-output data. Now as the first model based design strategy is more applied, so we do much research on system identification to identify the plant model. The whole theory of system identification can be divided into four categories, i.e. experiment design, model structure selection, model parameter identification and model structure validation test. Further the more research is concerned on the first three categories. To be the author's knowledge, the study on model structure validation under closed loop condition is very little.

There are three common identification methods in closed loop identification, i.e. direct approach, indirect approach and joint input-output approach, where the feedback is neglected in direct approach and the plant model is identified directly using the input-output data. For the indirect approach, the feedback effect is considered and the input-output from the whole closed loop condition are used to identify the plant model. The joint input-output approach is very similar to indirect approach. In [1], three methods are presented to identify closed loop system. In [2], researches on the system identification theory are introduced in time domain. Similarly the frequency domain system identification is given in [3]. A new virtual closed loop method for closed loop identification is proposed in [4]. In [5], one projection algorithm is proposed on the basis of the prediction error recursive method. In [6], when many inputs exist in closed loop, whether can closed loop be identified with parts of the inputs controlled? The relationship between closed loop 
identification and closed loop control are obtained in [7]. In [8], the linear matrix inequality is used to describe the problem of optimal input design in closed loop. Further the least cost identification experiment problem is analyzed in [9]. The power spectral of the input signal is considered to be an objective function and the accuracy of the parameter estimations is the constraints [10]. In [11], $\mathrm{H}$ infinity norm from robust control is introduced to be the objective function in the optimal input design problem. Based on the $\mathrm{H}$ infinity norm, the uncertainty between the identified model and nominal model is measured and the optimal input is chosen by minimizing this uncertainty [12]. The selection of the optimal input can also be determined from the point of asymptotic behavior about the parameter estimation [13]. The Persistent excitation input in closed loop is analyzed and we obtain some conditions about how to obtain persistent excitation [14]. Reference [15] considers how to apply closed loop identification into adaptive control so that bias and covariance terms are isolated separately. All above results hold when the number of the observed signals will convergent to infinity.

There are little papers about model structure validation test now. Only in [2] and [15], model structure validation in open loop identification has been presented and the standard cross correlation test is proposed to test the confidence interval of the cross variance matrix between the prediction error and input from the probabilistic sense. Because of the simple structure of open loop, the process of deriving the covariance matrix is very easy. For model structure validation in engineering, the more effective strategy is to do one similar experiment again. After exciting the formal system with a group of new inputs, we compare if the actual output is consistent with the identified output. Although this test is simple, we can not analyze the accuracy and credibility of the identified model. In our paper [16], two probabilistic model uncertainties and optimum input filter are derived from some statistical properties of the parameter estimation. The probabilistic bounds and optimum input filter are based on an asymptotic normal distribution of the parameter estimator and its covariance matrix, which was estimated from sampled data. Using some results from our former paper [16], in this paper we continue to study the problem of model structure validation for closed loop system identification. So in order to reflect the identification accuracy, here we apply the statistical probability framework to derive the variance matrix of the unknown parameters firstly. This variance matrix is decomposed into one inter product form which is used to construct one uncertainty bound about the unknown parameter estimation. This uncertainty bound is called by confidence interval and it constitutes the guaranteed confidence region test with respect to the model parameter estimation under closed loop condition. Furthermore our aim is to find if the identified model is any good at describing the measured data and being a basis for latter controller design. To validate the effectively of the identified model, we construct one bound corresponding to the model error. This bound is proposed by an inequality condition, which connects the algebraic nature between the model error, the input signal, the model validation test quantity. Based on the bound and the proposed inequality condition, the problem of model structure validation for closed loop system is reformulated to verify if the absolute value of model error satisfies above inequality. Due to the closed loop system is considered here, some priori properties of closed loop system are provided to simplify the bound.

\section{Problem Description}

Consider the following actual closed loop system with output feedback (see Figure 1).

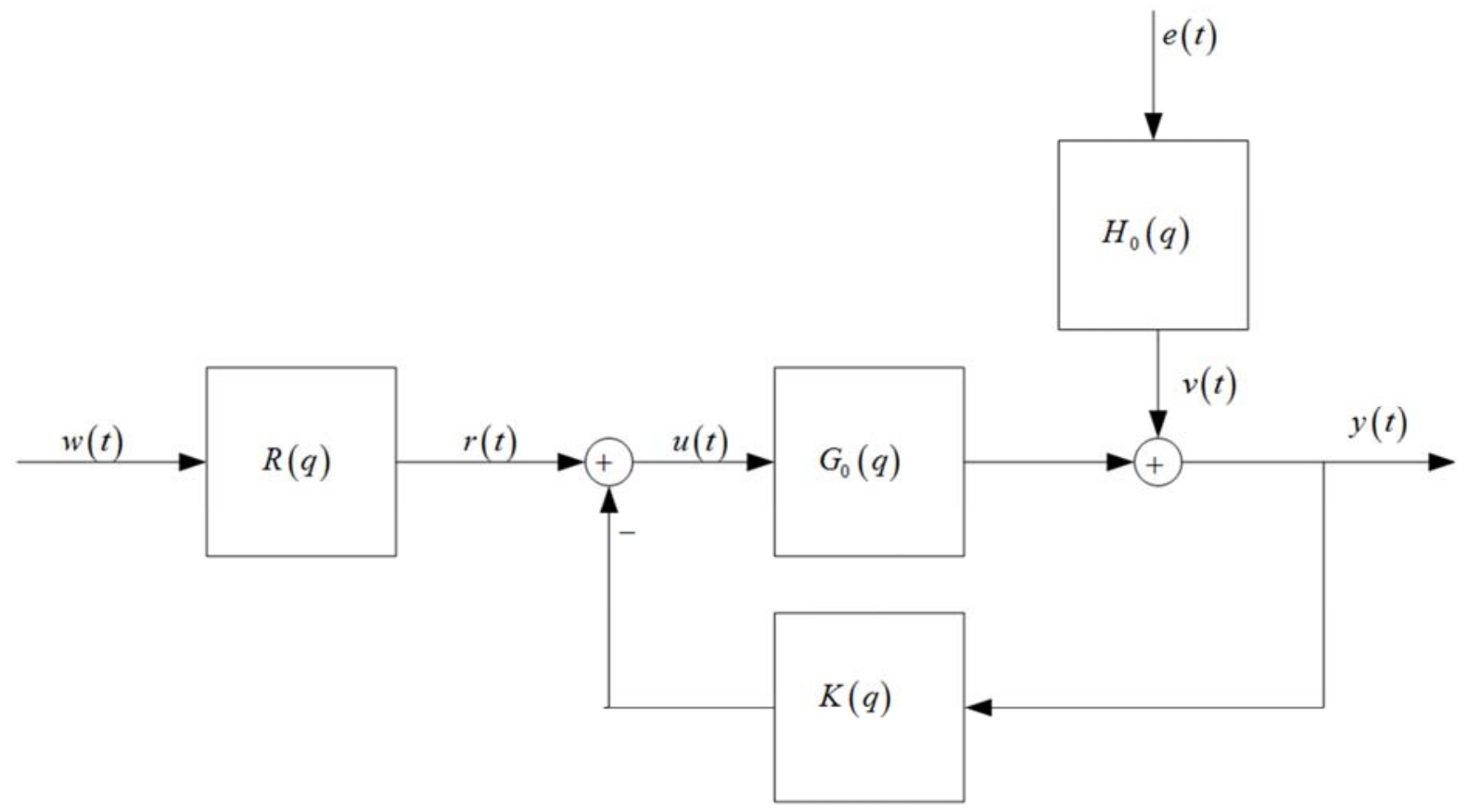

Figure 1. Structure of the closed loop system. 
where $G_{0}(q)$ is the true plant model; $H_{0}(q)$ is the noise filter, they are all linear time invariant transfer functions. $K(q)$ is a stable linear time invariant controller, here we assume this controller is priori known. The excited signal $r(t)$ and external disturbance $e(t)$ are assumed to be uncorrelated, $e(t)$ is a white noise with zero mean value and variance $\lambda_{0} \cdot v(t)$ is a colored noise which can be obtained by passing white noise $e(t)$ through the noise filter $H_{0}(q) \cdot u(t)$ and $y(t)$ are the input-output signals corresponding to plant model $G_{0}(q)$.

Rewriting excited signal $r(t)$ as white noise $w(t)$ passing through shaping filter $R(q) \cdot R(q)$ is the power spectrum factor with stable non-minimal phase of excited signal $r(t) \cdot q$ is the delay operator, it means that $q u(t)=u(t+1)$.

As $r(t)=R(q) w(t)$, the power spectrum density of excited signal is given as

$$
\phi_{r}(\omega)=R(q) R^{*}(q) \phi_{w}(\omega)=|R(q)|^{2}
$$

In closed loop system structure, through some computations, we derive the transfer function form

$$
y(t)=G_{0}(q) R(q) w(t)-G_{0}(q) K(q) y(t)+H_{0}(q) e(t)
$$

Continuing to do some computations and we get

$$
\begin{gathered}
y(t)=\frac{G_{0}(q) R(q)}{1+G_{0}(q) K(q)} w(t)+\frac{H_{0}(q)}{1+G_{0}(q) K(q)} e(t) \quad \begin{array}{c}
\text { denotes the number of total obse } \\
\text { According to }(3), \text { the pred } \\
\text { calculated as the one step ahead }
\end{array} \\
\begin{aligned}
u(t)=\frac{R(q)}{1+G_{0}(q) K(q)} w(t)-\frac{K(q) H_{0}(q)}{1+G_{0}(q) K(q)} e(t) & (2) \\
\hat{y}(t, \theta) & =\frac{1+G(q, \theta) K(q)}{H(q, \theta)} \times \frac{G(q, \theta) R(q)}{1+G(q, \theta) K(q)} w(t) \\
+\left[1-\frac{1+G(q, \theta) K(q)}{H(q, \theta)}\right] y(t) & \\
= & \frac{G(q, \theta) R(q)}{H(q, \theta)} w(t)+\frac{H(q, \theta)-1-G(q, \theta) K(q)}{H(q, \theta)} y(t)
\end{aligned}
\end{gathered}
$$

To simplify the analysis process, define the sensitivity function as.

$$
S_{0}(q)=\frac{1}{1+G_{0}(q) K(q)}
$$

The output of closed loop system can be written as.

$$
y(t)=G_{0}(q) R(q) S_{0}(q) w(t)+H_{0}(q) S_{0}(q) e(t)
$$

As now our goal is not emphasized on identification method, but on the model structure validation, so the only simple direct approach is used to describe the basic ideas.

\section{Confidence Region Test of Model Parameter}

Introduce the unknown parameter vector in closed loop system, the parameterized form given by.

$$
y(t, \theta)=\frac{G(q, \theta) R(q)}{1+G(q, \theta) K(q)} w(t)+\frac{H(q, \theta)}{1+G(q, \theta) K(q)} e(t)
$$

where $\theta$ denotes the unknown parameter vector, it exists in the parameterized plant model $G(q, \theta)$ and noise model $H(q, \theta)$ respectively. The goal of closed loop identification is to identify the unknown parameter vector $\hat{\theta}_{N}$ from one given input-output data set $Z^{N}=\{y(t), u(t)\}_{t=1}^{N}$, where $N$ denotes the number of total observed data.

According to (3), the prediction of $y(t, \theta)$ can be calculated as the one step ahead prediction.

Computing the one step ahead prediction error or residual, now it becomes.

$$
\begin{aligned}
& \varepsilon(t, \theta)=y(t)-\hat{y}(t, \theta) \\
& \quad=\frac{1+G(q, \theta) K(q)}{H(q, \theta)}\left[y(t)-\frac{G(q, \theta) R(q)}{1+G(q, \theta) K(q)} w(t)\right]
\end{aligned}
$$

In the standard prediction error algorithm, when using the input-output data $Z^{N}=\{y(t), u(t)\}_{t=1}^{N}$ with the data number $N$, the parameter vector is identified by.

$$
\hat{\theta}_{N}=\underset{\theta}{\arg \min } V_{N}\left(\theta, Z^{N}\right)=\underset{\theta}{\arg \min } \frac{1}{N} \sum_{t=1}^{N} \varepsilon^{2}(t, \theta)
$$


Defining the asymptotic limit parameter estimate $\theta^{*}$ as

$$
\theta^{*}=\underset{\theta}{\arg \min } \lim _{N \rightarrow \infty} E\left\{V_{N}\left(\theta, Z^{N}\right)\right\}
$$

where $E$ denotes the expectation operator. In the common identification process, assume that there always exists one true parameter vector $\theta_{0}$ such that.

$$
G\left(q, \theta_{0}\right)=G_{0}(q), H\left(q, \theta_{0}\right)=H_{0}(q)
$$

This assumption shows that the identified model is contained in the considered model set. Based on some results from reference [2], we get the asymptotic matrix of the parameter estimate.

$$
\operatorname{cov} \hat{\theta}_{N}=\lambda_{0}\langle\varphi, \varphi\rangle^{-1}
$$

where $\langle\varphi, \varphi\rangle$ denotes some inter product operator, $\varphi$ is the negative gradient of the predictor error, i.e it can be computed from:

$$
\varphi(t, \theta)=-\frac{\partial \varepsilon(t, \theta)}{\partial \theta}=\frac{\partial \hat{y}(t, \theta)}{\partial \theta}
$$

Next we give the calculation process of the negative gradient of the predictor error under closed loop condition below. As (7) is a basic formula in studying asymptotic analysis, we combine (3) and (4) to get.

$$
\begin{aligned}
\hat{y}(t, \theta) & =\frac{G(q, \theta) R(q)}{H(q, \theta)} w(t) \\
+ & \frac{H(q, \theta)-1-G(q, \theta) K(q)}{1+G(q, \theta) K(q)} R(q) w(t) \\
+ & \frac{H(q, \theta)-1-G(q, \theta) K(q)}{1+G(q, \theta) K(q)} e(t) \\
= & \frac{G(q, \theta) R(q)}{1+G(q, \theta) K(q)} w(t) \\
& +\frac{H(q, \theta)-1-G(q, \theta) K(q)}{1+G(q, \theta) K(q)} e(t)
\end{aligned}
$$

Substituting (8) into (5) and computing the partial derivative operations with respect to unknown parameter vector $\theta$, and then we have

$$
\begin{aligned}
& \frac{\partial \varepsilon(t, \theta)}{\partial \theta}=\frac{G^{\prime}(\theta) K H(\theta)-[1+G(\theta) K] H^{\prime}(\theta)}{H(\theta)} \\
& \quad \times \frac{1}{1+G(\theta) K} e(t)-\frac{1}{H(\theta)} \\
& \quad \times \frac{G^{\prime}(\theta) R[1+G(\theta) K]-G(\theta) R G^{\prime}(\theta) K}{1+G(\theta) K} w(t) \\
& =\left[\frac{G^{\prime}(\theta) K}{H(\theta)[1+G(\theta) K]}-\frac{H^{\prime}(\theta)}{H(\theta)}\right] e(t) \\
& \quad-\left[\frac{G^{\prime}(\theta) R}{H(\theta)}-\frac{G(\theta) R G^{\prime}(\theta) K}{H(\theta)[1+G(\theta) K]}\right] w(t)
\end{aligned}
$$

where $G^{\prime}(\theta)$ denotes the partial derivative operation with respect to $\theta$, and the delay operator $q$ are all ignored to simply the derivations.

Using the uncorrelated assumption between white noise $e(t)$ and $w(t)$, i.e. it holds that

$$
E e(t) w^{T}(t)=0
$$

Putting ahead one $H(\theta)$ in (9), and rewriting it as.

$$
\begin{aligned}
& \frac{\partial \varepsilon(t, \theta)}{\partial \theta}=\frac{1}{H(\theta)} \\
& \quad \times\left(\begin{array}{l}
{\left[G^{\prime}(\theta) K S(\theta)-H^{\prime}(\theta)\right] e(t)} \\
\quad-\left[G^{\prime}(\theta) R-G(\theta) R G^{\prime}(\theta) S(\theta) K\right] w(t)
\end{array}\right)
\end{aligned}
$$

where we use the parameterized sensitivity function

$$
S(\theta)=\frac{1}{1+G(\theta) K}
$$

As the following equality holds

$$
R-G(\theta) R K S(\theta)=\frac{R}{1+G(\theta) K}=R S(\theta)
$$

Rewriting (10) as the following matrix form

$$
\begin{aligned}
& \frac{\partial \varepsilon(t, \theta)}{\partial \theta}=\frac{1}{H(\theta)} \\
& \times\left[\begin{array}{ll}
G^{\prime}(\theta) & H^{\prime}(\theta)
\end{array}\right]\left[\begin{array}{cc}
K S(\theta) H(\theta) & R S(\theta) \\
-1 & 0
\end{array}\right]\left[\begin{array}{l}
e(t) \\
w(t)
\end{array}\right]
\end{aligned}
$$

According to (7), the asymptotic covariance matrix is that

$$
\begin{aligned}
& P_{\theta}=\operatorname{cov} \hat{\theta}_{N}=\lambda_{0}\left[E \varphi\left(t, \theta_{0}\right) \varphi^{T}\left(t, \theta_{0}\right)\right]^{-1}=\langle\phi, \phi\rangle^{-1} \\
& \phi=\frac{1}{\sqrt{\lambda_{0}} H_{0}}\left[\begin{array}{ll}
G^{\prime}\left(\theta_{0}\right) & H^{\prime}\left(\theta_{0}\right)
\end{array}\right]\left[\begin{array}{cc}
\sqrt{\lambda_{0}} K S_{0} H_{0} & R S_{0} \\
-\sqrt{\lambda_{0}} & 0
\end{array}\right]
\end{aligned}
$$

where $G^{\prime}(\theta)$ and $H^{\prime}(\theta)$ are given as respectively

$$
G^{\prime}(\theta)=\frac{\partial G(\theta)}{\partial \theta}, \quad H^{\prime}(\theta)=\frac{\partial H(\theta)}{\partial \theta}
$$

On basis of (12), we have the asymptotic result

$$
\hat{\theta}_{N} \stackrel{N \rightarrow \infty}{\longrightarrow} \theta_{0}
$$

It shows that the parameter estimator $\hat{\theta}_{N}$ will converge to its limit $\theta_{0}$, and further $\hat{\theta}_{N}$ will asymptotically converge $(N \rightarrow \infty)$ to normally distributed random variable with mean $\theta_{0}$ and variance $P_{\theta}$.

$$
\sqrt{N}\left(\hat{\theta}_{N}-\theta_{0}\right) \rightarrow \mathbb{N}\left(0, P_{\theta}\right) \text {, as } N \rightarrow \infty
$$


This asymptotic result can be rewritten in a quadratic form, and then we get one $\lambda^{2}$ distribution.

$$
N\left(\hat{\theta}_{N}-\theta_{0}\right)^{T} P_{\theta}^{-1}\left(\hat{\theta}_{N}-\theta_{0}\right) \stackrel{N \rightarrow \infty}{\longrightarrow} \lambda_{n}^{2}
$$

where $n$ is the number of degrees of freedom in the $\lambda^{2}$ distribution, being equal to the dimension of the parameter vector. Equation (13) implies that the random variable $\hat{\theta}_{N}$ satisfies one uncertainty bound.

$$
\begin{aligned}
& \hat{\theta}_{N} \in D\left(\alpha, \theta_{0}\right) \\
& D\left(\alpha, \theta_{0}\right)=\left\{\theta_{0} / N\left(\theta-\theta_{0}\right)^{T} P_{\theta}^{-1}\left(\theta-\theta_{0}\right) \leq \lambda_{n, \alpha}^{2}\right\}
\end{aligned}
$$

with $\lambda_{n, \alpha}^{2}$ corresponding to a probability level $\alpha$ in $\lambda_{n}^{2}$ distribution, But now in order to quantity the uncertainty on $\theta_{0}$ rather than on $\hat{\theta}_{N}$. For every realization of $\hat{\theta}_{N}$, it holds that

$$
\hat{\theta}_{N} \in D\left(\alpha, \theta_{0}\right) \Leftrightarrow \theta_{0} \in D\left(\alpha, \hat{\theta}_{N}\right)
$$

It signifies that

$$
\begin{aligned}
& \theta_{0} \in D\left(\alpha, \hat{\theta}_{N}\right) \text { with probability } \alpha \\
& D\left(\alpha, \hat{\theta}_{N}\right)=\left\{\theta / N\left(\hat{\theta}_{N}-\theta\right)^{T} P_{\theta}^{-1}\left(\hat{\theta}_{N}-\theta\right) \leq \lambda_{n, \alpha}^{2}\right\}
\end{aligned}
$$

Equations (14) and (15) give the confidence intervals of unknown parameter estimator under closed loop condition. The probability level of the event $\hat{\theta}_{N} \in D\left(\alpha, \theta_{0}\right)$ holds is at least $\alpha$.

\section{One Bound on Model Error}

A new technique for estimating bias and variance contributions to the model error is suggested in this section. Then model structure validation procedure gives a direct measurement of the model error of closed loop system, so the problem of model structure validation for closed loop system identification can be changed to test whether the model error obeys one inequality condition. Our problem is to figure out if identified model $G\left(\hat{\theta}_{N}\right)$ is any good at describing the measured data and to give a statement how far away the model might be from a true description.

As it is very useful to consider the prediction error or model residual, we could write model residual as follows based on equation (5).

$$
\begin{aligned}
& \varepsilon(t, \theta)=\frac{1+G(\theta) K}{H(\theta)} \\
& \times\left[\frac{G_{0} R}{1+G_{0} K} w(t)+\frac{H_{0}}{1+G_{0} K} e(t)-\frac{G(\theta) R}{1+G(\theta) K} w(t)\right]
\end{aligned}
$$

Inserting the parameter estimator $\hat{\theta}_{N}$ into the predictor error $\varepsilon(t, \theta)$, we get.

$$
\begin{aligned}
& \varepsilon\left(t, \hat{\theta}_{N}\right)=\frac{1+G\left(\hat{\theta}_{N}\right) K}{H\left(\hat{\theta}_{N}\right)} \\
& \times\left[\frac{G_{0} R}{1+G_{0} K} w(t)-\frac{G\left(\theta^{*}\right) R}{1+G\left(\theta^{*}\right) K} w(t)+\frac{G\left(\theta^{*}\right) R}{1+G\left(\theta^{*}\right) K} w(t)\right. \\
& \left.-\frac{G\left(\hat{\theta}_{N}\right) R}{1+G\left(\hat{\theta}_{N}\right) K} w(t)+\frac{H_{0}}{1+G_{0} K} e(t)\right] \\
& =\underbrace{\frac{1+G\left(\hat{\theta}_{N}\right) K}{H\left(\hat{\theta}_{N}\right)}\left[\frac{G_{0} R}{1+G_{0} K}-\frac{G\left(\theta^{*}\right) R}{1+G\left(\theta^{*}\right) K}\right] w(t)}_{A_{1}\left(t, G_{0}, \theta^{*}\right)} \\
& +\underbrace{\frac{1+G\left(\hat{\theta}_{N}\right) K}{H\left(\hat{\theta}_{N}\right)}\left[\frac{G\left(\theta^{*}\right) R}{1+G\left(\theta^{*}\right) K}-\frac{G\left(\hat{\theta}_{N}\right) R}{1+G\left(\hat{\theta}_{N}\right) K}\right] w(t)}_{A_{2}\left(t, \hat{\theta}_{N}, \theta^{*}\right)} \\
& +\underbrace{\frac{1+G\left(\hat{\theta}_{N}\right) K}{H\left(\hat{\theta}_{N}\right)} \frac{H_{0}}{1+G_{0} K} e(t)}_{A_{3}\left(t, \hat{\theta}_{N}, G_{0}, H_{0}\right)}
\end{aligned}
$$

Remark:

(a) the first term $A_{1}\left(t, G_{0}, H_{0}, \theta^{*}\right)$ of equation (17) is the residual signal part induced by the asymptotic bias of estimated model $G\left(\theta^{*}\right)$.

(b) the second term $A_{2}\left(t, \hat{\theta}_{N}, \theta^{*}\right)$ is also the residual signal part but induced by the variance error of the parameter estimator $G\left(\hat{\theta}_{N}\right)$.

(c) the third term $A_{3}\left(t, \hat{\theta}_{N}, G_{0}, H_{0}\right)$ is the effect of measurement noise $e(t)$, and represents the un-modeling errors in the estimated noise model.

Then the model structure validation problem is formulated into one hypothesis testing problem.

$$
\gamma_{0}: A_{1}\left(t, G_{0}, H_{0}, \theta^{*}\right)=0
$$

Under the condition that the input signal is persistent excitation, then condition of the above hypothesis testing holds is that.

$$
\frac{G\left(\theta^{*}\right)}{1+G\left(\theta^{*}\right) K}=\frac{G_{0}}{1+G_{0} K} \Rightarrow \theta^{*}=\theta_{0}
$$


When hypothesis testing problem $\gamma_{0}$ holds, then the residual signal $\varepsilon\left(t, \hat{\theta}_{N}\right)$ contains only two terms

$$
\varepsilon_{1}\left(t, \hat{\theta}_{N}\right)=A_{2}\left(t, \hat{\theta}_{N}, \theta^{*}\right)+A_{3}\left(t, \hat{\theta}_{N}, G_{0}, H_{0}\right)
$$

To give a detailed expression on model residual $\varepsilon_{1}\left(t, \hat{\theta}_{N}\right)$, we continue to rewrite it.

$$
\begin{aligned}
\varepsilon_{1}\left(t, \hat{\theta}_{N}\right)=A_{2}\left(t, \hat{\theta}_{N}, \theta^{*}\right)+A_{3}\left(t, \hat{\theta}_{N}, G_{0}, H_{0}\right) & =\frac{1+G\left(\hat{\theta}_{N}\right) K}{H\left(\hat{\theta}_{N}\right)}\left[\frac{G\left(\theta^{*}\right)}{1+G\left(\theta^{*}\right) K}-\frac{G\left(\hat{\theta}_{N}\right)}{1+G\left(\hat{\theta}_{N}\right) K}\right] r(t) \\
& +\frac{1+G\left(\hat{\theta}_{N}\right) K}{H\left(\hat{\theta}_{N}\right)} \frac{H_{0}}{1+G_{0} K} e(t) \\
= & \frac{1+G\left(\hat{\theta}_{N}\right) K}{H\left(\hat{\theta}_{N}\right)} \frac{1}{1+G\left(\theta^{*}\right) K} \tilde{G} r(t) \\
& \underbrace{\frac{1+G\left(\hat{\theta}_{N}\right) K}{H\left(\hat{\theta}_{N}\right)} \frac{H_{0}}{1+G_{0} K} e(t)}_{L_{1}(q)} \\
= & \underbrace{L_{1}(q) \tilde{G} r(t)+\tilde{L}_{2}(q) e(t)}_{L_{2}(q)}
\end{aligned}
$$

In derivation process of equation (20), we use two known equalities

$$
\left\{\begin{array}{l}
r(t)=R(q) w(t) \\
\tilde{G}(q)=G\left(\theta^{*}\right)-G\left(\hat{\theta}_{N}\right)
\end{array}\right.
$$

By observing equation (20), now the problem is that, what can be said about model error $\tilde{G}(q)$ based on the information in $Z^{N}=\{y(t), u(t)\}_{t=1}^{N}$ ? Ideally we want to give one bound on $\left|\tilde{G}\left(e^{i w}\right)\right|$ valid for each frequency $w$. Before giving our main technical result of one bound on model error, we define some preliminaries as basis for our next derivation process.

Then input-output data record corresponding to the whole closed loop system is that

$$
Z^{N}=\{y(t), r(t)\}_{t=1}^{N}
$$

The periodogram of the input sequence $\{r(t), t=1,2 \cdots N\}$ is defined by

$$
\left|R_{N}(w)\right|^{2}=\frac{1}{N}\left|\sum_{t=1}^{N} r(t) e^{-i w t}\right|^{2}
$$

Let regression vector $\varphi(t)$ be that

$$
\varphi(t)=\left[\begin{array}{llll}
r(t) & r(t-1) & \cdots & r(t-M+1)
\end{array}\right]^{T}
$$

where $M$ is the delay time in regression vector, and its autocorrelation function is defined as

$$
R_{N}=\frac{1}{N} \sum_{t=1}^{N} \varphi(t) \varphi^{T}(t)
$$

A scalar measurement of the correlation between past input and model residual is constructed

$$
\tilde{\xi}_{N}^{M}=\frac{1}{N}\left\|\sum_{t=1}^{N} \varphi(t) \varepsilon_{1}(t)\right\|_{R_{N}^{-1}}^{2}
$$

Here $\|$.$\| denotes the common Euclidian norm, such as$

$$
\|v\|_{R}=v^{*} R v
$$

The procedure to obtain one bound on model error lies in the following theorem.

Theorem 1: Assume that the input-output data record $Z^{N}=\{y(t), r(t)\}_{t=1}^{N}$ is subject to equation (20), let $G\left(\hat{\theta}_{N}\right)$ be an identified model, and construct measure $\tilde{\xi}_{N}^{M}$ from (22)-(25). Assume that there exists a $\delta$, such that $R_{N}>\delta I$, then the model error $\tilde{G}(q)$ satisfied the following inequality

$$
\begin{aligned}
& {\left[\left.\frac{1}{2 \pi} \int_{-\pi}^{\pi}\left|L_{1}\left(e^{i w}\right)\right|^{2}\left|\tilde{G}\left(e^{i w}\right)\right|^{2}\left|R_{N}\left(e^{i w}\right)\right|\right|^{2} d w\right]^{\frac{1}{2}}} \\
& \quad \leq(1+\eta)\left[\frac{1}{N} \tilde{\xi}_{N}^{M}\right]^{\frac{1}{2}}+(1+\eta) x_{N}+(2+\eta) C_{r} \sum_{k=M}^{\infty}\left|\rho_{k}\right|
\end{aligned}
$$

Here

$$
x_{N}=\left\|\frac{1}{N} \sum_{t=1}^{N} L_{2}(q) e(t) \varphi(t)\right\|_{R_{N}^{-1}} ;
$$

$\rho_{k}$ is the impulse response of $L_{1}(q) \tilde{G}(q)$;

$\left|R_{N}(w)\right|^{2}$ is the periodogram of the input sequence;

$$
\begin{gathered}
C_{r}=\max _{1 \leq t \leq N}|r(t)| ; \\
\eta=\frac{C_{r} M}{\sqrt{N \delta}}
\end{gathered}
$$

By the appropriate choice of feedback controller $K(q)$, the size of model error over arbitrarily small frequency intervals can be arbitrarily small. From above equation (26), 
we see that if one identified model $G\left(\hat{\theta}_{N}\right)$ can be used for the next control process, then the absolute value of model error must satisfy this inequality condition.

Before giving an explicit proof about inequality condition (26), one important lemma from [2] is used here.

Lemma: Let

$$
\begin{aligned}
& w(t)=N(q) u(t)=\sum_{k=0}^{\infty} n_{k} u(t-k) \\
& \varphi(t)=\left[\begin{array}{llll}
u(t) & u(t-1) & \cdots & u(t-M+1)
\end{array}\right]^{T} \\
& R_{N}=\frac{1}{N} \sum_{t=1}^{N} \varphi(t) \varphi^{T}(t) \\
& U_{N}(w)=\frac{1}{\sqrt{N}} \sum_{t=1}^{N} u(t) e^{-i w t}
\end{aligned}
$$

Assume that

$$
\begin{aligned}
& |u(t)| \leq C_{u} \quad t=1,2 \cdots N \\
& R_{N}>\delta I
\end{aligned}
$$

Let

$$
\tilde{\rho}_{M}=\sum_{k=M}^{\infty}\left|n_{k}\right|
$$

And define that

$$
\begin{aligned}
& \beta^{2}=\left\|\frac{1}{N} \sum_{t=1}^{N} w(t) \varphi_{1}(t)\right\|_{R_{N}^{-1}}^{2} \\
& B^{2}=\frac{1}{2 \pi} \int_{-\pi}^{\pi}\left|N\left(e^{i w}\right)\right|^{2}\left|U_{N}(w)\right|^{2} d w
\end{aligned}
$$

Then for $\eta=\frac{C_{r} M}{\sqrt{N \delta}}$, we have that.

$$
B \leq(1+\eta) \beta+(2+\eta) C_{u} \tilde{\rho}_{M}
$$

Based on this lemma, now we give a short proof about Theorem 1.

Proof: As

$$
\tilde{G}(q)=G\left(\theta^{*}\right)-G\left(\hat{\theta}_{N}\right)
$$

and

$$
\varepsilon_{1}\left(t, \hat{\theta}_{N}\right)=L_{1}(q) \tilde{G} r(t)+L_{2}(q) e(t)
$$

Thus

$$
\begin{aligned}
& {\left[\frac{1}{N} \tilde{\xi}_{N}^{M}\right]^{\frac{1}{2}}=\left\|\frac{1}{N} \sum_{t=1}^{N} \varphi(t) \varepsilon_{1}(t)\right\|_{R_{N}^{-1}}} \\
& \quad=\left\|\frac{1}{N} \sum_{t=1}^{N}\left(L_{1}(q) \tilde{G}(q) r(t)+L_{2}(q) e(t)\right) \varphi(t)\right\|_{R_{N}^{-1}} \\
& \quad \geq \gamma-x_{N}
\end{aligned}
$$

where

$$
\gamma=\left\|\frac{1}{N} \sum_{t=1}^{N} L_{1}(q) \tilde{G}(q) r(t) \varphi(t)\right\|_{R_{N}^{-1}}
$$

Then

$$
\gamma \leq\left[\frac{1}{N} \tilde{\xi}_{N}^{M}\right]^{\frac{1}{2}}+x_{N}
$$

Using lemma, we see that

$$
\begin{aligned}
& {\left[\frac{1}{2 \pi} \int_{-\pi}^{\pi}\left|L_{1}\left(e^{i w}\right)\right|^{2}\left|\tilde{G}\left(e^{i w}\right)\right|^{2}\left|R_{N}\left(e^{i w}\right)\right|^{2} d w\right]^{\frac{1}{2}}} \\
& \quad \leq(1+\eta) \gamma+(2+\eta) C_{r} \tilde{\rho}_{M}
\end{aligned}
$$

Substituting (29) into (30), then inequality condition (26) is proved.

As closed loop system is considered here, then some properties of closed loop system can be used to simplify that inequality condition using the assumption of that $e(t)$ is uncorrelated with $w(t)$.

$$
\operatorname{Er}(t) e(t)=E R(q) w(t) e(t)=0
$$

We compute the first term in $\left[\frac{1}{N} \tilde{\xi}_{N}^{M}\right]^{\frac{1}{2}}$ as follows.

$$
\begin{aligned}
& \left\|\frac{1}{N} \sum_{t=1}^{N} L_{2}(q) e(t) \varphi(t)\right\|_{R_{N}^{-1}}=L_{2}(q) r_{r e}^{T} R_{N}^{-1} r_{r e} L_{2}(q) \\
& =L_{2}(q)\left[\begin{array}{llll}
r_{r e}(0) & r_{r e}(1) & \cdots & r_{r e}(M-1)
\end{array}\right] \\
& \times R_{N}^{-1}\left[\begin{array}{c}
r_{r e}(0) \\
r_{r e}(1) \\
\vdots \\
r_{r e}(M-1)
\end{array}\right] L_{2}(q)=0
\end{aligned}
$$

Then the second term in $\left[\frac{1}{N} \tilde{\xi}_{N}^{M}\right]^{\frac{1}{2}}$ is also computed. 


$$
\begin{aligned}
& \left\|\frac{1}{N} \sum_{t=1}^{N} L_{1}(q) \tilde{G}(q) r(t) \varphi(t)\right\|_{R_{N}^{-1}} \\
& =L_{1}(q) \tilde{G}(q)\left[\begin{array}{llll}
r_{r}(0) & r_{r}(1) & \cdots & r_{r}(M-1)
\end{array}\right] \\
& \times R_{N}^{-1}\left[\begin{array}{c}
r_{r}(0) \\
r_{r}(1) \\
\vdots \\
r_{r}(M-1)
\end{array}\right] L_{1}(q) \tilde{G}(q) \\
& =\left|L_{1}(q) \tilde{G}(q)\right|^{2}\left[|R(q)|^{2} \quad 0 \quad \cdots \quad c\right] R_{N}^{-1}\left[\begin{array}{c}
|R(q)|^{2} \\
0 \\
\vdots \\
0
\end{array}\right] \\
& =\left|L_{1}(q) \tilde{G}(q)\right|^{2}|R(q)|^{4}\left[\begin{array}{llll}
1 & 0 & \cdots & 0
\end{array}\right] R_{N}^{-1}\left[\begin{array}{c}
1 \\
0 \\
\vdots \\
0
\end{array}\right]
\end{aligned}
$$

where

$$
\left\{\begin{array}{l}
r_{r e}(\tau)=\frac{1}{\sqrt{N}} \sum_{t=1}^{N} r(t) e(t-\tau) \\
r_{r}(\tau)=\frac{1}{\sqrt{N}} \sum_{t=1}^{N} r(t) r(t-\tau)
\end{array}\right.
$$

Before substituting equation (31) and (32) into (26), we should obtain the $(1,1)$ element of matrix $R_{N}$. Observing the explicit structure of regression vector $\varphi(t)$ in matrix $R_{N}$, we have that.

$$
\varphi(t)=R(q)\left[\begin{array}{llll}
w(t) & w(t-1) & \cdots & w(t-M+1)
\end{array}\right]^{T}
$$

and

$$
R_{N}=\frac{1}{N} \sum_{t=1}^{N} \varphi(t) \varphi^{T}(t)=|R(q)|^{2} \frac{1}{N} N=|R(q)|^{2}
$$

The above derivation process corresponding to matrix $R_{N}$ is very easy, for example we only give one equality

$$
\begin{aligned}
& \varphi(1) \varphi^{T}(1)=|R(q)|^{2}\left[\begin{array}{c}
w(1) \\
w(0) \\
\vdots \\
w(-M+1)
\end{array}\right] \\
& \times\left[\begin{array}{llll}
w(1) & w(0) & \cdots & w(-M+1)
\end{array}\right]^{T} \\
& =|R(q)|^{2} I
\end{aligned}
$$

So for all $t=1,2 \cdots N$, we have that.

$$
\varphi(t) \varphi^{T}(t)=|R(q)|^{2} I, \quad t=1,2 \cdots N
$$

Substituting (33) into (32), we get that

$$
\left\|\frac{1}{N} \sum_{t=1}^{N} L_{1}(q) \tilde{G}(q) r(t) \varphi(t)\right\|_{R_{N}^{-1}}=\left|L_{1}(q) \tilde{G}(q)\right|^{2}|R(q)|^{2}
$$

Combing equation (31) and (35), the inequality condition in Theorem 1 can be simplified as follows

$$
\begin{aligned}
& {\left[\frac{1}{2 \pi} \int_{-\pi}^{\pi}\left|L_{1}\left(e^{i w}\right)\right|^{2}\left|\tilde{G}\left(e^{i w}\right)\right|^{2}\left|R_{N}\left(e^{i w}\right)\right|^{2} d w\right]^{\frac{1}{2}}} \\
& \leq(1+\eta)\left|L_{1}\left(e^{i w}\right)\right|\left|\tilde{G}\left(e^{i w}\right)\right|\left|R\left(e^{i w}\right)\right|+(2+\eta) C_{r} \sum_{k=M}^{\infty}\left|\rho_{k}\right|
\end{aligned}
$$

where variable $q$ is changed to variable $e^{i w}$.

From equation (36), the identification model $G\left(\hat{\theta}_{N}\right)$ is useful on the condition that the model error $\tilde{G}(q)$ must obey one bound, which is described by inequality condition (36). So the problem of model structure validation for closed loop system identification is reformulated to ver.

Ify if the absolute value of model error satisfies above inequality.

\section{Simulation Example}

To prove the model structure validation strategies under closed loop condition, we consider one simulation system.

$$
\begin{aligned}
y(t) & =G_{0}(q) R(q) w(t)-G_{0}(q) K(q) y(t) \\
& +H_{0}(q) e(t)
\end{aligned}
$$

Where $G_{0}(q), H_{0}(q)$ and $K(q)$ are assumed to be as follows respectively.

$$
\begin{aligned}
& G_{0}(q)= \\
& \quad \frac{0.01293 q^{-1}+0.1062 q^{-2}+0.1058 q^{-3}+0.01279 q^{-4}}{1-0.2482 q^{-1}+1.091 q^{-2}-0.2441 q^{-3}+0.9822 q^{-4}} \\
& H_{0}(q)=1, \quad K(q)=1
\end{aligned}
$$

Here the noise model is 1 and it shows the external disturbance acting on closed loop reduces to the white noise disturbance. The feedback is the common positive feedback. The noise $e(t)$ is a white noise with zero mean and unit variance, input $w(t)$ is similar to noise $e(t)$. In order to analyze the confidence region of the model parameter and cross correlation function, we set shaping filter as $R(q)=1$.

Choose the number of observed data set $\{y(t), w(t)\}_{t=1}^{N}$ as $N=500$, and apply direct approach to identify the unknown parameters in parameterized plant model $G_{0}(q)$. The qualities of nine model parameters affect the output response directly. So the identification accuracy or credibility of model parameters can be all measured by the output 
response of closed loop.

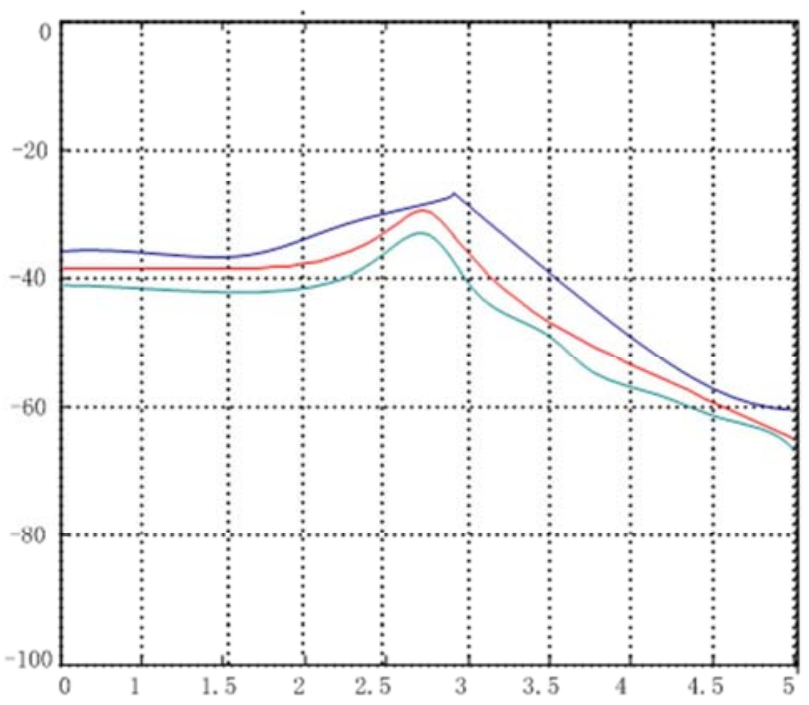

Figure 2. Confidence interval of amplitude in Bode plot.

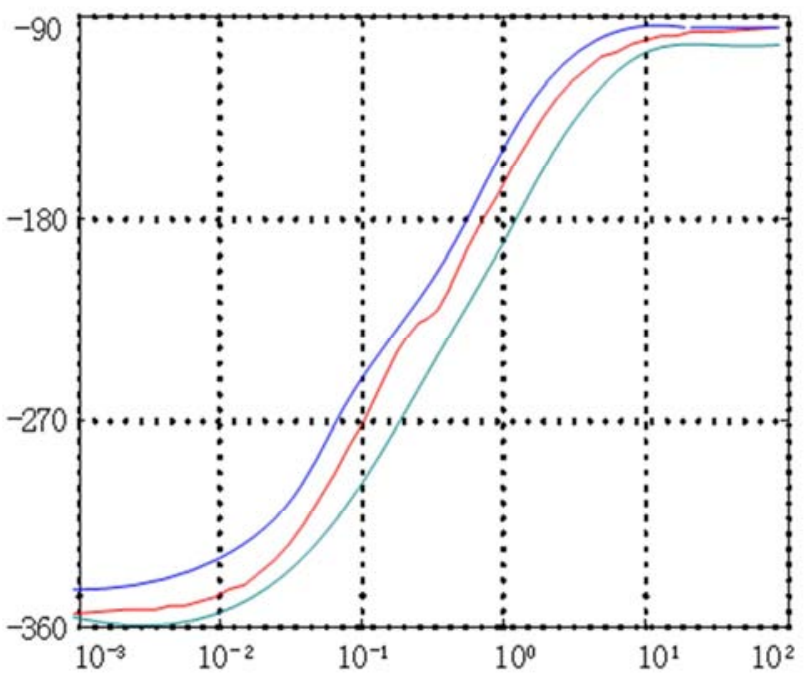

Figure 3. Confidence interval of phase in Bode plot.

The whole output frequency response curves are showed in Figure 2, based on estimated model parameters. The red curve is the actual true amplitude curve from Bode plot tool. When the estimated model parameters are contained in the uncertainty bound with probability level 0.99 , the amplitude curves lie above or low the red curve. From Figure 1, we see these three curves are very close and the red amplitude curve lies between two confidence amplitude curves with probability level 0.99 .

As using Matlab simulation tool to simulate the output response of Bode plot in closed loop, the phase plot is get with amplitude plot simultaneously. The confidence interval phase plot is given in Figure 3, and the red phase curve lies also between two confidence phase curves with the probability level 0.99 . This is similar to the derivation of Figure 2.

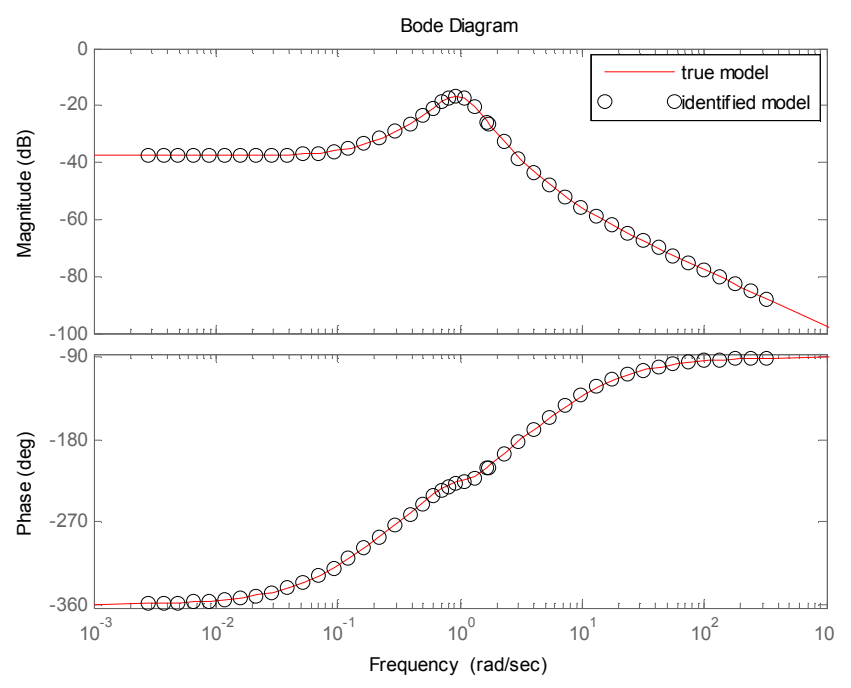

Figure 4. Comparing of the true model and its identified model.

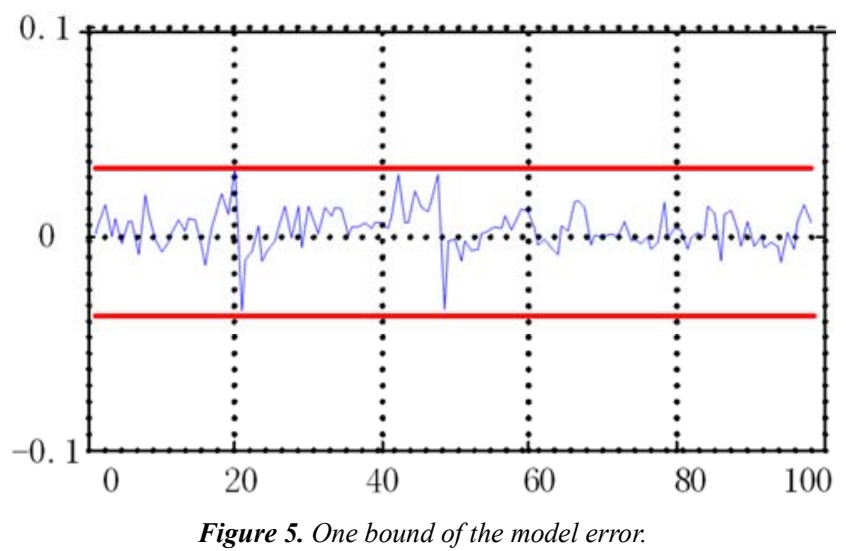

To verify the efficiency of the identified model $G\left(\hat{\theta}_{N}\right)$ and make sure that this identified model can be used to replace the true model, we compare the Bode responses through true model $G_{0}(q)$ and its identified model $G\left(\hat{\theta}_{N}\right)$ respectively in Figure 4. From Figure 4, we see that these two Bode response curves coincide with each other, it means that the model error $\tilde{G}(q)$ will converge to zero with time increases in Figure 5, then this model error can be neglected in our next process in designing controllers.

\section{Conclusion}

In this paper we analyze the model structure validation problem for closed loop condition from two aspects: (1) confidence region of model parameter, (2) one bound on the model error.

This bound tells us that if one appropriate feedback controller is chosen, the model error will converge to zero. So this bound can guide us to design controllers with two-degrees of freedom. The next subject is to construct one virtual variable to reformulate this asymptotic variance matrix as one projection form of this virtual variable along some vector space. 


\section{References}

[1] Urban Forssel, Lennart Ljung, "Closed loop identification revisted," Automatica, vol. 35, no. 7, pp. 1215-1241, 1999.

[2] Ljung, L, "System identification: Theory for the user," Prentice Hall, 1999.

[3] Pintelon R, Schoukens J, "System identification: a frequency domain approach," New York: IEEE Press, 2001.

[4] Juan C, Augero, "A virtual closed loop method for closed loop identification," Automatica, vol 47, no. 8, pp. 1626-1637, 2011.

[5] U, Forssell, L Ljung, "Some results on optimal experiment design," Automatica, vol 36, no. 5, pp. 749-756, 2000.

[6] M, Leskers, "Closed loop identification of multivariable process with part of the inputs controlled," International Journal of Control, vol 80, no. 10, pp. 1552-1561, 2007.

[7] Hakan Hjalmarssion, "From experiment design to closed loop control," Automatica, vol 41, no. 3, pp. 393-438, 2005.

[8] Hakan Hjalmarssion, "Closed loop experiment design for linear time invariant dynamical systems via LMI," Automatica, vol 44, no. 3, pp. 623-636, 2008.

[9] X, Bombois, "Least costly identification experiment for control," Automatica, vol 42, no. 10, pp. 1651-1662, 2006.

[10] Roland Hildebrand, "Identification for control: optimal input design with respect to a worst case gap cost function," SIAM Journal of Control Optimization, vol 41, no. 5, pp. 1586-1608, 2003.

[11] M, Gevers, "Identification of multi input systems: variance analysis and input design issues," Automatica, vol 42, no. 410, pp. 559-572, 2006.

[12] M, Gevers, "Identification and information matrix: how to get just sufficiently rich," IEEE Transactions on Automatic control, vol 54, no. 12, pp. 2828-2840, 2009.

[13] Graham C Goodin, "Bias issues in closed loop identification with application to adaptive control," Communications in Information and Systems, vol 2, no. 4, pp. 349-370, 2002.

[14] James S Welsh, "Finite sample properties of indirect nonparametric closed loop identification," IEEE Transactions on Automatic control, vol 47, no. 8, pp. 1277-1291, 2002.

[15] Sippe G Douma, "Validity of the standard cross correlation test for model structure validation," Automatica, vol 44, no. 4, pp. 1285-1294, 2008.

[16] Wang Jian-hong, Wang Yan-xiang. "Model structure validation for closed loop system identification," International Journal of Modelling, Identification and Control, vol 27, no. 4, pp. 323-331, 2017. 